\title{
Norois
}

Environnement, aménagement, société

$222 \mid 2012$

Xynthia

\section{Après Xynthia : vers un repli stratégique et un État fort?}

Denis Mercier

\section{OpenEdition}

1 Journals

Édition électronique

URL : http://journals.openedition.org/norois/3826

DOI : $10.4000 /$ norois.3826

ISBN : 978-2-7535-1843-8

ISSN : 1760-8546

Éditeur

Presses universitaires de Rennes

Édition imprimée

Date de publication : 28 février 2012

Pagination : 7-9

ISBN : 978-2-7535-1815-5

ISSN : 0029-182X

\section{Référence électronique}

Denis Mercier, « Après Xynthia : vers un repli stratégique et un État fort? », Norois [En ligne], 222 | 2012

mis en ligne le 30 mars 2012, consulté le 31 mars 2021. URL : http://journals.openedition.org/norois/ 3826 ; DOl : https://doi.org/10.4000/norois.3826

\section{(c) Tous droits réservés}




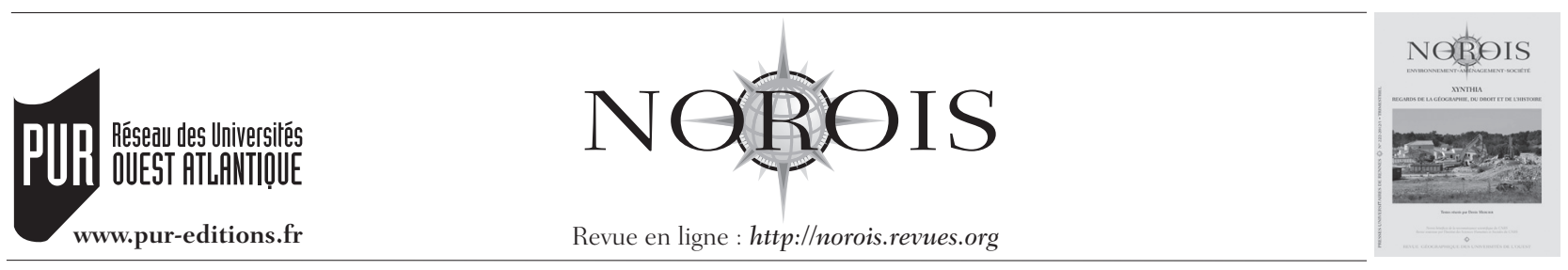

\title{
Éditorial \\ Après Xynthia : vers un repli stratégique et un État fort?
}

\author{
Denis Mercier
}

Professeur de géographie à l'université de Nantes (IGARUN)

Directeur du laboratoire Géolittomer (CNRS - UMR 6554 LETG)

Membre de l'Institut Universitaire de France

"Le mitage de l'espace agricole et des dunes s'étend chaque année. Sur toute la façade méridionale du littoral, le contrôle strict des affectations des sols est urgent si l'on ne veut pas voir s'étendre un tissu de banlieue pavillonnaire anarchique qui pose déjà de redoutables problèmes d'aménagement et de cô̂t pour les collectivités locales. Il faut protéger, délimiter et aider les zones conchylicoles et de cultures spécialisées. Or, on peut craindre que les populations, dont les activités essentielles sont désormais tournées vers l'exploitation du tourisme, ce que la composition des conseils municipanx reflète, prennent conscience trop tardivement des dangers du laisser-faire. »

\section{Alain Chauvet et Jean Renard, 1978. La Vendée. Le pays. Les hommes, Les Sables-d'Olonne, Éditions du Cercle-d'Or, p. 181.}

Plus de trois décennies se sont écoulées depuis que ces lignes ont été écrites par deux géographes nantais à propos des communes littorales du sud de la Vendée, tragiquement touchées dans la nuit du 27 au 28 février 2010 par la tempête Xynthia. Les craintes exprimées alors prennent aujourd'hui un relief particulier puisque toutes les victimes de ces communes habitaient dans des résidences construites récemment : la dernière conséquence du « laisser-faire », évoqué jadis, en matière de politique d'aménagement du littoral.

Cette tempête Xynthia a profondément marqué les esprits, puisqu'avec 41 victimes, elle représente un des événements les plus meurtriers survenus en France ces dernières décennies (Vinet, 2010). Elle pose des questions de nature sur un phénomène météo-marin bien connu par ailleurs, les «submersions de tempêtes » (Caspar et al., 2007). Au-delà de ce cas d'école de submersion marine, cet exemple interroge la politique d'aménagement du littoral français et plus largement celle de l'ensemble des zones inondables du territoire.

Ce numéro n'aborde pas les effets morphogéniques des tempêtes puisque la revue Norois a consacré un numéro thématique sur cette question récemment (Suanez, 2010). Les inondations littorales s'observent sur les côtes basses lorsqu'une dépression atmosphérique, générant une forte agitation du plan d'eau (vague) et une onde de tempête inversement proportionnelle à la baisse de la pression atmosphérique (une baisse d'un hectopascal induit une élévation d'un centimètre du plan d'eau) se conjugue dans le temps avec des fortes marées et conduit à une surcote. La tempête Xynthia estelle exceptionnelle? La réponse donnée par l'étude des géographes Vinet et Feuillet et leurs collabora- 
teurs et des historiens Sarrazin, Acerra et Sauzeau, est négative et sans appel. Le réchauffement climatique peut-il induire une augmentation de ce genre de phénomène? En fréquence? En intensité? L'élévation du niveau marin envisagée d'ici à la fin du $\mathrm{XXI}^{\mathrm{e}}$ siècle va-t-il profondément changer la donne? Les réponses sont plus délicates car le consensus n'est pas scientifiquement acquis, mais laissent à penser que Xynthia ne sera pas sans réplique dans les décennies à venir.

Cette tempête Xynthia repose surtout deux grandes séries de questions à la société.

La première est devenue une question centrale dans la politique française : comment réduire la vulnérabilité des biens et des personnes? Les questions de développement des territoires inondables doivent intégrer l'exposition des populations à des dangers mortels, c'est-à-dire des inondations rapides comme c'est le cas des submersions marines ou des crues éclairs du domaine méditerranéen. Les critères pour définir ces zones « noires » sont discutées par Mercier et Chadenas.

La seconde relève plus de la gestion des risques au sens large. Suite à la tempête Xynthia, des maisons ont été détruites, amorçant un processus de retrait des zones dangereuses, mais nos sociétés contemporaines ne sont pas toujours prêtes à céder des territoires à la nature. Les plans de prévisions des risques (PPR) sont par ailleurs insuffisants et parfois mal adaptés aux enjeux. Le plan communal de sauvegarde (PCS), imposé par la loi du 13 août 2004, est loin d'être adopté par les collectivités. La question de la culture du risque partagée par tous (État, élus, populations) est de nouveau posée. Faut-il remettre en cause la loi du 13 juillet 1982, qui repose sur la solidarité nationale pour des risques connus et dont l'exposition est évitable, quand on connaît les effets pervers et déresponsabilisant de ce système assurantiel? Rehausser les digues est-elle la solution unique? Là aussi la réponse est négative et sans appel. Les digues ont été progressivement édifiées pour soustraire des espaces productifs par la saliculture et l'agriculture aux caprices de la mer. Elles étaient souvent détruites comme nous le montre l'article du médiéviste J.-L. Sarrazin. Elles ont été édifiées ni pour protéger des maisons principales ni surtout des résidences secondaires construites récemment sur un littoral convoité. Vivre en zone submersible : à quel prix? Le prix à payer en termes de vie humaine, en termes de coûts d'infrastructures pour la société. Quel est le coût de l'inaction et du laisser-faire? Quel serait le coût d'une politique de dépoldérisation du littoral?

Xynthia montre surtout que l'intérêt général n'a pas prévalu sur l'intérêt particulier, que la privatisation des bénéfices de la manne foncière l'emporte et qu'en revanche, la collectivisation des coûts (par le biais de la solidarité nationale, les indemnisations de l'État, des collectivités territoriales, des assurances...), permet aux bénéficiaires des terrains de rester en zone à risque. Assisterait-on à la même politique de «colonisation » du littoral, si la reconstruction des digues ne revenait pas à la collectivité, mais aux particuliers qui en bénéficient?

Xynhtia est aussi l'occasion de faire le procès de la décentralisation et du désengagement de l'État. L'échelle communale montre bien des faiblesses en termes d'attribution de la rente foncière comme le souligne le travail du juriste P.-Y. Legal. Le recours à la domanialisation serait une solution. Les textes de loi existent, comme nous le rappelle l'article de P. Le Louarn, encore faut-il une volonté de promouvoir l'acceptabilité de la loi. Les PPR littoraux sont encore insuffisamment prescrits et l'article R 111-2 du code de l'urbanisme qui donne la possibilité de refuser l'implantation d'enjeux même en l'absence d'un PPR approuvé, peu utilisé.

Cette tempête pose des questions scientifiques multiples. Elles posent aussi clairement la nécessité d'une approche pluridisciplinaire associant les spécialistes des approches spatiales (géographes), temporelles (historiens) et des spécialistes du droit, ce que ce numéro illustre. Cependant, d'autres élargissements disciplinaires sont nécessaires pour appréhender ces questions d'aménagement des littoraux. Les économistes possèdent des outils robustes d'analyses coûts-bénéfices notamment qui permettent de répondre à des questions centrales, comme par exemple le maintien ou la délocalisation des infrastructures en zone d'aléa fort. Les psychologues et les sociologues peuvent permettrent de comprendre pourquoi les personnes acceptent de vivre dans des zones dangereuses voire même refusent d'en partir après une tragédie comme Xynthia. Les spécialistes de l'altimétrie spatiale peuvent, grâce aux nouvelles données LIDAR fournir des cartes 
précises. Les questions centrales au cœur de ce débat reviennent à des questions d'échelles. Que ce soit pour définir des zones inondables, des zones à risques mortels, des intensités de tempête, des évacuations, tout est question d'échelle, d'indicateurs, de seuils. Les scientifiques peuvent apporter des éléments de réponse et doivent aussi être à l'écoute des questionnements posés par les acteurs de l'aménagement du territoire (les politiques, les services de l'État, les personnes en charge de la sécurité des biens et des personnes sur le territoire, des victimes des inondations...). Où des inondations par submersion marine peuvent-elles survenir dans les années à venir ? À partir de quelle intensité de vent, de hauteur de houle, de coefficient de marée, faut-il faire évacuer les populations? Suite à Xynthia, les services de Météo-France ont accéléré leur travail de prévision pour le littoral français et proposent, depuis octobre 2011, des cartes de vigilance vaguesubmersion. Ces cartes ont été pour la première fois éditées au moment de la tempête Joachim du 16 décembre 2011 et une cinquantaine d'habitants de la commune de Saint-Hilaire-de-Riez en Vendée a été évacué à titre préventif.

Puisse cette tempête faire changer les mentalités, inverser les tendances pour amorcer un repli stratégique, là où c'est nécessaire et possible, permettre à l'État de reprendre la main sur des dossiers fondamentaux comme celui de l'aménagement du territoire, notamment dans des zones dangereuses pour la vie des populations, en imposant l'intérêt général et une hauteur de vue, une autorité, une détermination, une légitimité et une responsabilité assumée pour des perspectives à long terme. Puisse Xynthia permettre la fin du déni, de la cécité, de l'amnésie et de la cupidité dans le développement des territoires, notamment littoraux.

\section{Bibliographie}

Caspar R., Costa S., Jakob E., 2007. Fronts froids et submersions de tempête dans le nord-ouest de la France, La Météorologie, 57 , p. 37-47.

SuAnEz S. (dir.), 2010. Impacts morphogéniques des tempêtes. Les enseignements de la tempête du 28 mars 2008, Norois, $215,146 \mathrm{p}$.

Vinet F., 2010. Le risque inondation. Diagnostic et gestion, Paris, Lavoisier, 318 p. 